\title{
Intervenção da equoterapia no equilíbrio estático de criança com síndrome de Down
}

\author{
Equotherapy intervention in the static balance in a child with Down syndrome
}

\section{Cristiane Helita Zorél Meneghetti ${ }^{1}$, Carlos Henrique da Silva Porto ${ }^{2}$, Cristina Iwabe ${ }^{3}$, Sofia Poletti ${ }^{4}$}

\section{RESUMO}

Objetivo. Verificar a influência da equoterapia no equilíbrio estático em uma criança com Síndrome de Down. Método. Foi realizado um estudo de caso em uma criança com Síndrome de Down, gênero masculino, 9 anos de idade. $O$ participante foi filmado na vista anterior (plano frontal) e perfil (plano sagital) nas condições com visão e sem visão. Na filmagem na condição de olhos fechados, foi utilizado um óculos de natação totalmente vedado, com a finalidade do participante não ter nenhuma informação visual. O instrumento utilizado foi a Biofotogrametria Computadorizada que serviu como referência angular para verificar as oscilações do corpo em equilíbrio estático. A intervenção da Equoterapia foi realizada durante 16 sessões, uma vez por semana de equoterapia, empregando as técnicas de equitação e atividades eqüestres. Resultados. Os graus de oscilações avaliados antes e depois da intervenção da equoterapia apresentaram diminuição de oscilações nos plano frontal e sagital. Conclusão. O presente estudo mostrou que com a intervenção da equoterapia, a criança com Síndrome de Down apresentou melhora em seu equilíbrio estático

Unitermos. Equilíbrio Postural, Síndrome de Down, Fotogrametria. Citação. Meneghetti CHZ, Porto CHS, Iwabe C, Poletti S. Intervenção da equoterapia no equilíbrio estático de criança com síndrome de Down.

Trabalho Realizado no Centro Universitário Hermínio Ometto, UNIARARAS, Araras-SP, Brasil.

1.Fisioterapeuta, Mestre em Distúrbios do Desenvolvimento, MACKENZIE, São Paulo-SP, Brasil.

2.Fisioterapeuta, Especialista em neurologia adulto e infantil, UNIARARAS, Araras-SP, Brasil.

3.Fisioterapeuta, Doutoranda em Ciências Médicas, UNICAMP, Campinas-SP, Brasil.

4.Fisioterapeuta, Docente da UNIARARAS, Araras-SP, Brasil.

\section{SUMMARY}

Objective. Verify the influence of hippotherapy in the static balance of one child with Down syndrome. Method. It was done a study of case in one child with Down syndrome, male, nine years old. The subject was filmed in anterior (frontal view) and profile (sagittal view) views in these two conditions: with and without vision. During the video takes with the eyes closed, it was used a swimming goggle completely sealed, with the purpose of not allowing the subject to have any visual information. The selected pictures were quantified by Computerized Biophotogrammetry, which served as angular reference to verify body's oscillations in the static balance. It was done 16 hippotherapy sessions, once a week, using equitation techniques and equestrians activities, to provide physical, psychological, educational and social benefits. Results. The degrees of oscillation evaluated before and after the hippotherapy intervention showed lower values in the frontal and sagittal views. Conclusion. This study showed that the intervention with hippotherapy provided improvement in the static balance in this child with Down syndrome.

Keywords. Postural Balance, Down Syndrome, Photogrammetry.

Citation. Meneghetti CHZ, Porto CHS, Iwabe C, Poletti S. Equotherapy intervention in the static balance in a child with Down syndrome.

Endereço para correspondência:

Cristiane HZ Meneghetti

Rua das Nogueiras, 95

CEP 13601-291, Araras-SP, Brasil.

E-mail crismeneghetti@yahoo.com.br

Relato de Caso

Recebido em: 08/07/2008

Revisado em: 09/07/2008 a 13/08/2008

Aceito em: 14/08/2008

Conflito de interesses: não 


\section{INTRODUÇÃO}

A Síndrome de Down (SD) foi descrita clinicamente pela primeira vez pelo médico inglês John Langdon Down em 1866, identificando sua etiologia genética apenas em 1959, com o francês Jerome'.

Essa síndrome é causada por alterações cromossômicas envolvendo o par do cromossomo 21, o qual se apresenta em trissomia, resultando em alterações físicas e mentais ${ }^{1,2}$.

Na pesquisa sobre os aspectos do desenvolvimento infantil na SD, observa-se que muitos têm seu foco nos fatores que influenciam nas aquisições motoras da criança, demonstrando-se que estas apresentam um atraso significante no desenvolvimento de habilidades motoras e no controle postural, indicando que estes marcos emergem em tempo diferenciado das crianças com desenvolvimento típico ${ }^{3-6}$.

Muitas crianças com SD sentam e levantam sem apoio aproximadamente 10 meses mais tarde do que as crianças neurologicamente normais ${ }^{7}$.

Alguns aspectos vêm sendo sugeridos como causa do atraso na aquisição dos marcos motores como a fraqueza exacerbada nas articulações, fraqueza muscular e hipotonia são as causas principais dessas diferenças ${ }^{6,8-11}$.

Uma das possíveis causas dessas desordens de movimento deve-se a hipoplasia do cerebelo, responsável pela hipotonia muscular e associação deteriorada entre músculos sinérgicos, como visto nas reações posturais pré-programadas seguintes a perturbações ${ }^{9,11}$.

Segundo estudos prévios ${ }^{8,10,12}$, a falta de controle motor adequado nos indivíduos com SD, decorrentes das alterações cerebelares, contribui também para o atraso na aquisição dos marcos motores.

Outra disfunção observada na SD é o déficit do controle postural, relacionada com dificuldade na coordenação motora, integração sensória - motora. Os movimentos parecem desajeitados quando os indivíduos são lentos em se adaptar à tarefa, as condições do ambiente mutáveis ou são menos capazes de fazer ajustes posturais antecipatórios ${ }^{13}$.

Cavalgar se constitui em um processo de controle postural, além de proporcionar a sensação de independência e aumento da autoconfiança ${ }^{14,15}$.

Na equoterapia há a participação do corpo inteiro do praticante, contribuindo em seu desen- volvimento global ${ }^{14,15}$. O praticante adquire um posicionamento que inibe alguns padrões patológicos e com o cavalo ao passo recebe inúmeros estímulos que chegam ao Sistema Nervoso Central ${ }^{15}$.

Os estímulos mais importantes recebidos pelo praticante de equoterapia com o cavalo ao passo são: regularização tônica, coordenação motora, ritmo, flexibilidade, fortalecimento muscular e sistema respiratório ${ }^{14,15}$.

Entre os instrumentos de avaliação do equilíbrio encontra-se a Biofotogrametria Computadorizada, que se fundamenta na aplicação do princípio fotogramétrico às imagens fotográficas, obtidas de movimentos corporais. A essas imagens foram aplicadas bases de fotointerpretação, gerando se uma nova ferramenta de estudo da cinemática ${ }^{16,17}$.

Dessa forma, o objetivo do estudo foi verificar a influência da equoterapia no equilíbrio estático em uma criança com Síndrome de Down.

\section{MÉTODO \\ Relato de Caso}

Tratou-se de um modelo de estudo de caso, que obteve aprovação do Comitê de Ética e Mérito em Pesquisa sob o parecer $n^{\circ} 809 / 2007$ do Centro Universitário Hermínio Ometto - UNIARARAS.

Participou do estudo uma criança com Síndrome de Down, gênero masculino, nove anos de idade. Antes de iniciar a avaliação, o responsável assinou o Termo de Consentimento Livre e Esclarecido.

Primeiramente foi realizada uma avaliação do equilíbrio estático no laboratório de Biofotogrametria Computadorizada na clínica escola de fisioterapia da Uniararas.

Foi colocado no participante um marcador na região da glabela para avaliação na vista anterior e sobre o eurio para avaliação em perfil. Durante a filmagem o participante foi orientado a manter uma postura relaxada, com os braços posicionados ao lado do corpo o mais estável possível, e os pés paralelos sobre uma superfície plana, previamente marcada para os apoios plantares, tendo ao fundo um fio de prumo, que serviu de referência para a análise do ângulo.

O participante foi filmado na vista anterior (plano frontal) e na vista de perfil direito (plano sagital).

Na filmagem na condição de olhos abertos foi pedido ao participante que fixasse para um 
alvo formado por um papel amarelo em formato circular, posicionado na parede frontal da sala na altura dos olhos. Na filmagem na condição de olhos fechados, foi utilizado um óculos de natação totalmente vedado, com a finalidade do participante não ter nenhuma informação visual.

O participante foi posicionado de modo que os pontos antropométricos previamente marcados com ponto adesivo coincidissem com o fio de prumo, na vista anterior (glabela) como no perfil direito (eurio). Para a formação do ângulo, foi traçada uma reta ao vértice, perpendicular ao fio de prumo para determinação do ponto de interseção (Figuras 1 e 2).

A câmera filmadora foi posicionada sobre um tripé com prumo de superfície e em nível, a uma distância de 2,70 metros e a uma altura de 1,00 metros do solo. O tempo de exposição nas filmagens foi de 30 segundos para cada postura nas duas condições, com e sem visão.

Para análise das oscilações do corpo em equilíbrio estático, foi utilizado a Biofotogrametria Computadorizada como instrumento quantificador angular.

A fim de se obter o momento de maior oscilação ântero-posterior (plano sagital) na condição com visão e sem visão, e latero-lateral (plano frontal) na condição com visão e sem visão, as imagens foram analisadas quadro a quadro pelo programa Windows Movie Maker, e no momento de maior oscilação de cada plano a imagem foi selecionada e analisada pelo software Corel Draw, através da.
Biofotogrametria Computadorizada, determinando-se os valores angulares de maneira precisa e fidedigna

Após a avaliação iniciou-se a intervenção baseado na Equoterapia. As sessões foram realizadas no Centro Eqüestre Lucas Campagna, na cidade de Araras, São Paulo, utilizando-se um cavalo sem raça definida, 12 anos de idade, $310 \mathrm{~kg}$, $1,42 \mathrm{mts}$ de altura, três anos de treino e prática em Equoterapia.

Ao total foram realizadas 16 sessões uma vez por semana, durante 50 minutos. Cada sessão foi composta por alimentação do animal, escovação da pelagem, preparação para a montaria e montaria.

Destinaram-se as quatro primeiras sessões para a adaptação do praticante ao contato com o animal. A montaria no cavalo foi realizada sobre a manta, o celote com alça e com estribo no 14.

A pista de trabalho possui uma rampa de acesso, cujo terreno consiste em areia e plana, com largura de $\mathbf{2 0}$ metros e $\mathbf{4 0}$ metros de comprimento. A intervenção foi através do programa de hipoterapia e o percurso utilizado foi em formato de figuras geométricas.

Após a intervenção da equoterapia o participante realizou uma nova avaliação do equilíbrio estático.

Gráfico 1. Graus de oscilação em relação aos planos frontal e plano Sagital com e sem visão.

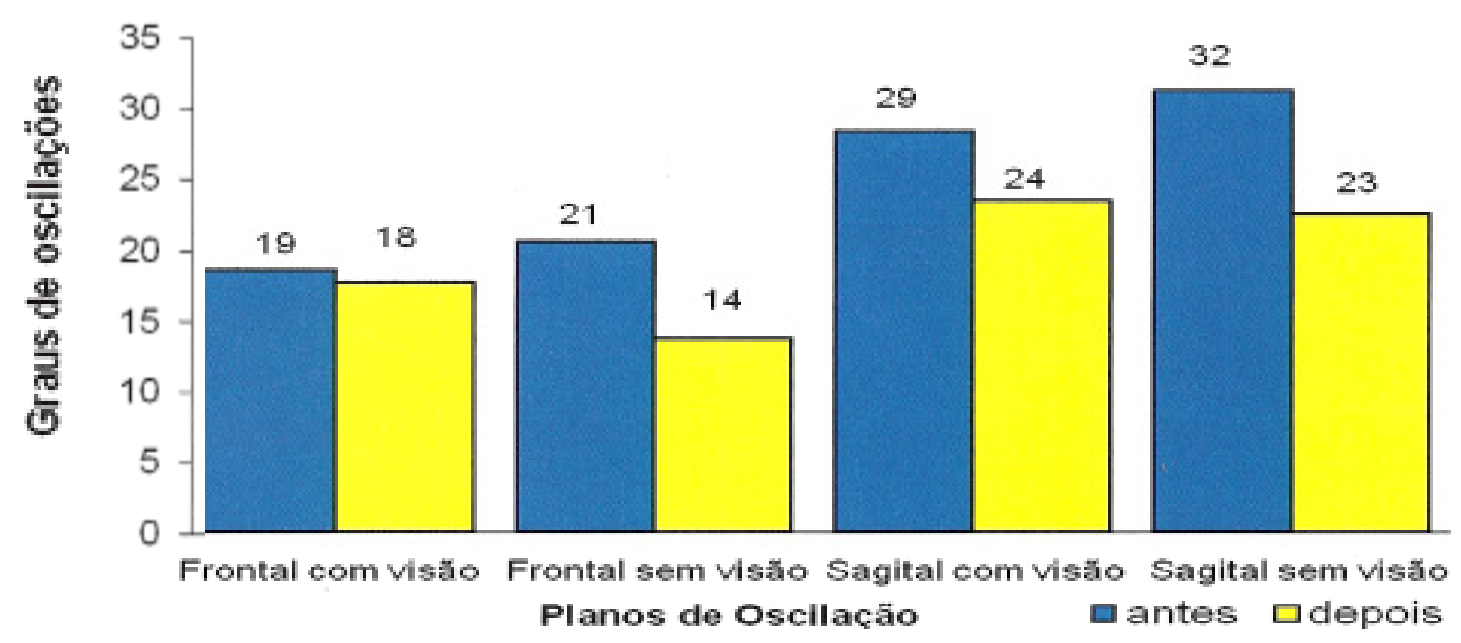




\section{RESULTADOS}

Verificou-se que os graus de oscilações avaliados depois da intervenção da equoterapia diminuíram tanto nos planos frontal como no sagital (Gráfico 1).

No plano frontal com visão, constatou-se uma redução de 19 para 18 graus, enquanto sem visão de 21 para 14 graus.

Da mesma forma, no plano sagital observou-se também uma diminuição dos graus de oscilação, sendo que com visão foi de 29 para 24 enquanto sem visão de 32 para 23 graus.

\section{DISCUSSÃO}

De maneira geral, verificou-se que com a intervenção da Equoterapia a criança com Síndrome de Down apresentou melhora em seu equilíbrio estático.

Nesse estudo o instrumento utilizado foi a Biofotogrametria Computadorizada que, além de ser um método fidedigno aos resultados apurados, de alta precisão e, conseqüentemente confiável, permite ainda registrar a imagem em arquivo, possibilitando comparação posterior e mensuração sempre que necessário ${ }^{16,17}$. Na avaliação do equilíbrio estático mostrou-se de fácil aplicação como evidenciado em vários estudos ${ }^{18-21}$.

O fato de crianças com SD oscilarem mais poderia ser decorrente da dificuldade para captar as informações sensoriais que determina a posição do corpo no espaço e a velocidade que o corpo está se movendo $22-24$. Isto parece ocorrer principalmente quando a informação proveniente de algum sistema sensorial é retirada ou manipulada, tornando-se maior a oscilação corporal entre indivíduos com $\mathrm{SD}^{13,23}$.

No processo de integração sensorial, as informações sensoriais são integradas pelo sistema de controle postural e pesos (valor de importância)

são dados a cada uma destas informações visual, vestibular e somatossensorial para tornar o controle postural um processo mais flexível, em função das constantes mudanças na relação do indivíduo com o ambiente ${ }^{25}$.

Com base nesta perspectiva, crianças com SD seriam menos eficientes na seleção e utilização de informações sensoriais adequadas ao contexto em que a tarefa é realizada.

$O$ resultado desse estudo mostrou que durante as tentativas em que a informação visual não estava disponível, a criança com SD apresentou maior oscilação, quando comparada com a condição onde a visão estava preservada.

Estudos apontam um aumento da oscilação corporal quando a informação sensorial é retirada ou manipulada ${ }^{26,27}$.

As experiências provocadas pelos movimentos do cavalo, pelo contato com o animal, associada a uma postura nova, pode estimular a potencialidade plástica do Sistema Nervoso Central por meio de estímulos sensitivos e motores, promovendo ao praticante o mesmo mecanismo perceptivo-cognitivo-motor ${ }^{14,15,28}$.

No entanto, o favorecimento de um maior controle motor, aumento do tônus muscular, a repetição do movimento, reações de equilíbrio e a percepção espaço-temporal dos vários segmentos corporais no espaço, explicaria as alterações observadas. Todavia, cabe ressaltar que o efeito da equoterapia é multifatorial, o que implica um conjunto de combinações e ajustes, contribuindo de maneira geral para o quadro do praticante ${ }^{14,15,28}$.

Contudo, neste estudo, os fatores de ajustes tônicos proporcionados pela equoterapia podem ter influenciado na melhora dos ajustes posturais, melhorando os graus de oscilações e consequentemente o equilíbrio.

\section{CONCLUSÃO}

A equoterapia, como atividade terapêutica, contribuiu para maior alinhamento biomecânico e consequentemente ativação e sinergia muscular adequada.

Esse controle muscular mais eficiente permitiu a otimização do equilíbrio da criança analisada. O recrutamento de maior número de indivíduos permitirá assim a confirmação dos nossos resultados, de modo a afirmar a influencia da equoterapia como método eficaz na aquisição e melhora do equilíbrio em crianças com SD.

\section{REFERÊNCIAS}

1.Nussbaum RL, Mcinnes RR, Willard HF. Genética Médica Thompson e Thompson. 6a. ed. Rio de Janeiro: Guanabara Koogan; 2002, 400p.

2.Moreira LMA, El-Hani C, Gusmão FAF. A síndrome de Down e sua patogênese: considerações sobre o determinismo genético. Rev Bras Psiquiatr 2000;22:96-9.

3.Wang WY, Ju YH. Promoting balance and jumping skills in children with Down syndrome. Percept Mot Skills 2002;94:4438.

4.Mancini MC, Silva PC, Gonçalves SC, Martins SM. Comparação do desempenho funcional de crianças portadoras de sín- 
drome de down e crianças com desenvolvimento normal aos 2 e 5 anos de idade. Arq Neuropsiquiatr 2003;61:409-15.

5.Pueschel S. Síndrome de Down - Guia Prático para pais e educadores. $9^{a}$ ed. Campinas: Papirus; 2005, 308p.

6.Polastri PF, Barela JA. Perception-Action Coupling in infants with Down syndrome: Effects of experience and Pratice. Adap Phys Act Quart 2005;22:39-56.

7.Marcondes E, Gonzales CH, Machado DVM, D'Agostinho G, Zuccolotto M, Sitian N. Crescimento normal e deficiente. $2^{\text {a }}$ ed.São Paulo: Savier; 1978, 179p.

8.Barros JF, Lemos SS, Soares MPS. Detecção de características específicas na articulação do joelho e do quadril que dificultam a marcha em indivíduos portadores de síndrome de down. Rev Alvorada 2003;1:41-64.

9.Vieregge P, Schulze-Rava H, Wessel K. Quantification of Postural Sway in Adult Down's syndrome. Develop Brain Dysf 1996;9:211-4.

10.Davis WE, Kelso JAS. Analysis of "Invariant Characteristics" the Motor Control of Down's syndrome and Normal Subjects. J Motor Beh 1982;14:194-212.

11.Kanode JO, Payne VG. Effects of variable practive on retention and motor schema development in Down syndrome subjects. Percept Mot Skills 1989;69:211-8.

12.Kleinhasns ACS, Silva MFMC. Processo cognitivo e plasticidade cerebral na síndrome de down. Rev Bras Educ Espec 2006;12:123-38.

13.Webber A, Virji-Babul N, Lesperance ER. Stiffiness and postural stability in adults with Down syndrome. Exp Brain Res 2004;155:450-8.

14.Medeiros M, Dias E. Equoterapia: Bases e Fundamentos. Rio de Janeiro: Revinter; 2002, 51p.

15.Cherng $\mathrm{R}$, Liao $\mathrm{H}$, Leung HWC, Hwang A. The effectiveness of therapeutic horseback riding in children with spastic cerebral palsy. Adapt Phys Activ Quart 2004; 21:103-21.

16.Ricieri DV. Validação de um protocolo de fotogrametria computadorizada e quantificação angular do movimento toracoabdominal durante a ventilação tranqüila (Dissertação). Uberlândia: Centro Universitário do Triângulo, 2000, 140p.

17.Ricieri DV. Biofotogrametria - Análise Cinemática Angular dos Movimentos. 2a ed. Curitiba: Revisada e Ampliada, 2005, 89p.
18.Baraúna MA, Duarte F, Sanchez HM, Canto RST, Maluá S, Silva $C D C$, et al. Avaliação do Equilíbrio Estático em Indivíduos Amputados de Membros Inferiores através da Biofotogrametria Computadorizada. Rev Bras Fisioter 2006;10:83-90.

19.Baraúna MA, Canto RST, Oliveira AS, Soares AB, Silva CDC Cardoso FAG. Avaliação do equilíbrio estático do portador de diabetes mellitus pela biofotogrametria. Diab Clin 2003;7:5762.

20.Baraúna MA, Barbosa SEM, Canto ST, Silva RAV, Silva CDC Baraúna KMP. Estudo do equilíbrio estático de idosos e sua correlação com quedas. Fisioter Bras 2004;5:136-41.

21.Guimarães EA. Avaliação do equilíbrio estático de indivíduos normais através da Biofotogrametria Computadorizada e da Oscilometria (Dissertação). Uberlândia: Centro Universitário do Triângulo; 2003.

22.Butterworth G, Cicchetti D. Visual calibration of posture in normal and motor retarded Down's syndrome infants. Percep 1978;7:513-25.

23.Kokubun M, Shinmyo T, Ogita M, Morita K, Furuta M, Haishi $\mathrm{H}$, et al. Comparison of postural control of children with downsyndrome and those with other forms of mental retardation. Percept Mot Skills 1987;84:499-504.

24.Vullerme N, Marin L, Debu B. Assessment of Static Postural Control in Teenagers with Down syndrome. Adap Phys Act Quart 2001;18:417-33.

25.Oie KS, Kiemel T, Jeka JJ. Multisensory fusion: simultaneous re-weighting of vision and touch for control of human posture. Cog Brain Res 2002;14:164-76.

26.Paulus WM, Straube A, Krafczyk S, Brandt T. Differential effects of retinal target displacement, changing size, and disparity in control of anterior posterior and lateral body sway. Exp Brain Res 1989;78:243-52.

27.Paulus WM, Straube A, Brandt T. Visual stabilization of posture: physiological stimulus characteristics and clinical aspects. Brain 1984;107:1143-63.

28.Krapivkin A, Nedashkovsky O, Khavkin A, Terent'eva I, Kolesnik L. Effect of intensive course of hipotherapy at children with cerebral palsy. Brain Dev 2001;23:189. 